\title{
Analytical Elastoplastic Solutions for Deep-buried Circular Tunnels Under Asymmetric Load
}

\author{
Zhang Xin ${ }^{1}$, Zhang Xiangping ${ }^{1}$, Wang Zhang ${ }^{2}$, Lang Min ${ }^{1}$, Zhao Suzhi ${ }^{1}$, Du Yuxiang ${ }^{2}$, \\ Sun Jinshan, ${ }^{2}$ * \\ ${ }^{1}$ China Railway 18 Bureau Group Co., Ltd, Tianjin, China \\ ${ }^{2}$ Hubei Key Laboratory of Blasting Engineering, Jianghan University, Wuhan, China \\ Email address: \\ 465723885@qq.com (Zhang Xin),52472106@qq.com (Zhang Xiangping),1326450079@qq.com (Wang Zhang), \\ Hollyghj@qq.com (Lang Min),sun99001@126.com (Zhao Suzhi),502469010@qq.com (Du Yuxiang), sunjinshan@cug.edu.cn (Sun Jinshan) \\ ${ }^{*}$ Corresponding author
}

\section{To cite this article:}

Zhang Xin, Zhang Xiangping, Wang Zhang, Lang Min, Zhao Suzhi, Du Yuxiang, Sun Jinshan. Analytical Elastoplastic Solutions for Deep-buried Circular Tunnels Under Asymmetric Load. American Journal of Civil Engineering. Vol. 9, No. 2, 2021, pp. 31-38.

doi: $10.11648 /$ j.ajce.20210902.11

Received: January 5, 2021; Accepted: April 1, 2021; Published: April 12, 2021

\begin{abstract}
The elastoplastic analysis of the surrounding rock of a circular tunnel is a very classic rock and soil mechanics problem. Previous scholars usually studied the situation under axisymmetric load, and they usually did not take the non-axisymmetric load distribution under the influence of ground stress and lateral pressure into account, which greatly affected its application in engineering practice. Approximate analytical solutions for calculating the plastic zone range, stress field and displacement field of the surrounding rock of a circular tunnel are inseparable from the consideration of the strength reduction and volumetric dilatancy characteristics of the rock material. The elastic-softening-residual plastic triple linear stress-strain model and the Mohr-Coulumb failure criterion are involved. The approximate analytical solutions of the residual stress field, strain field, displacement field and radius of the plastic zone in the elastic zone, plastic softening zone and surrounding plastic zone of the circular tunnel surrounding rock under axisymmetric load are deduced. The analytical solutions are valid only when the plastic zone is large and the lateral pressure coefficients $1 \leq \lambda<3$. The approximate analytical method is close to the calculation result of finite element method and can replace the finite element method to carry out simple elastic-plastic analysis of surrounding rock.
\end{abstract}

Keywords: Circular Tunnel, Asymmetrical Load, Strain Softening, Dilatancy, Elastoplastic Analysis

\section{Introduction}

The elastoplastic analysis of the surrounding rock of a circular tunnel is a classic rock and soil mechanics problem, which was put forward by R. Fenner and later corrected by $\mathrm{H}$. Kastner. Fenner and Kastner's research is based on an ideal elastoplastic medium, and mainly studied the situation under axisymmetric load. Many scholars at home and abroad subsequently proposed some calculation methods that consider the effects of surrounding rock strain softening and capacity expansion. For example, Mingjing Jiang proposed an analytical solution to the expansion problem of cylindrical holes based on the stress drop model and considering the dilatancy effect [1]. The approximate solution of the stress drop model in Hoek-Brown rock-soil medium was calculated by Sharan [2]. Yu considered the improvement of rock strength by the intermediate principal stress, and proposed a new unified strength theory [3]. Fan calculated the unified analytical solution of the surrounding rock of the circular cavern based on Yu's unified strength theory and the trilinear stress-strain softening model [4]. H. Kastner also gave an implicit approximate solution for the plastic zone distribution of surrounding rock under non-axisymmetric loads. Yu and Zheng revised H. Kastner's answer [5]. Sun introduced an approximate solution for the plastic zone distribution under non-axisymmetric loads [6]. Cai gave an analytical solution that takes the distribution of plastic zone in the loosening zone of the surrounding rock into account [7]. Park proposed the analytical solutions for the prediction of displacements 
around a circular opening in an elastic-brittle-plastic rock mass compatible with a linear Mohr-Coulomb or a nonlinear Hoek-Brown yield criterion [8]. Kargar proposed an analytical solution for isotropic, homogeneous, and visco-elastoplastic constitutive model of the surrounding rock of a circular tunnel under hydrostatic stress field [9]. Wang proposed the theoretical solution of stresses and displacements around a circular tunnel in Mohr Coulomb (M-C) rock mass considering axial in-situ stress, which can be used to calculate the case where the axial stress is not an intermediate stress [10]. Zhou proposed the elastic-plastic analytical solutions of elastic-plastic stress, strain and plastic zone of circular tunnel under different axial in-situ stresses based on the nonlinear Hoek Brown failure criterion [11]. Carranza described the relationship between the Hoek Brown parameter of rock mass strength and the mechanical response of underground caverns, and proposed a formula for describing the elastic-plastic behavior of rock by Hoek Brown criterion [12]. Jimenez proposed a new estimation method for equivalent Mohr Coulomb strength parameter, which can be used in the design of supporting tunnels in elastic-plastic rock masses satisfying the nonlinear empirical Hoek Brown failure criterion [13]. Sharan proposed a closed-form solution to calculate the displacement around a circular hole in brittle rock mass under hydrostatic stress field, which was found to be in an excellent agreement with that obtained by using the finite element method [14]. The elastic-plastic analytical methods of surrounding rock of circular tunnel are given based on different methods in the above-mentioned literatures. However, the coefficient of in-situ stress and lateral pressure of surrounding rock of circular tunnel has great influence on its plastic zone, stress field and displacement field. However, most of the existing analytical methods for elastic-plastic analysis of surrounding rock of circular tunnel are based on axisymmetric load distribution, the non axisymmetric load distribution under the influence of in-situ stress and lateral pressure is not well considered, which affects its application in engineering practice. $\mathrm{Lu}$ found an analytical solution to determine the plastic zone near a circular tunnel under non-axisymmetric stress based on the conformal transformation method of complex variable functions, but did not consider the strain softening phenomenon when the rock mass yielded [15].

Taking into account the influence of the lateral pressure coefficient of the in-situ stress on the plastic zone, stress field and displacement field of the surrounding rock, based on the trilinear stress-strain softening model, the Mohr-Coulomb yield criterion is used to derive approximate analytical solutions of the surrounding rock of a circular tunnel's stress field, strain field, displacement field and radius of plastic zone under non-axisymmetric load in this paper.

The remainder of this paper is organized as follows. In Section 2, the applicable rock mass constitutive model and yield criterion are determined, and the force model of the circular chamber is analyzed. In Section 3, the solution function of stress and displacement of surrounding rock in elastic zone under non-axisymmetric load is given. In Section
4, the solution function of stress and displacement of surrounding rock in plastic zone is given, and the extent of the plastic zone is calculated. In Section 5, through a calculation example, the analytical solution and the finite element calculation result are compared and verified, which shows the feasibility and accuracy of the method in this paper. Finally, conclusions are given in Section 6.

\section{Elastoplastic Analysis Mechanical Model of Circular Tunnel}

\subsection{Constitutive Model of Rock Mass and Yield Criterion}

Rock mechanics experiments show that the bearing capacity of most rock materials will decrease to some extent when the load reaches the limit value. At the same time, the volume of yield rock materials will generally increase with the increase of strain, that is, the strain softening and dilatancy effect of rock materials. Theoretical analysis and engineering practice show that the strain softening and dilatancy characteristics of geotechnical materials have a significant impact on the mechanical properties of surrounding rock. The mechanism of strain softening and dilatancy of geotechnical materials is very complex, Therefore, it is necessary to simplify the elastic-plastic analysis of circular cavern. The stress-strain relationship and the curves of $\varepsilon_{1}-\varepsilon_{3}$ used in this paper are shown in Figure 1 and Figure $2 \quad\left(\eta_{1}\right.$ and $\eta_{2}$ are expansion gradients, dimensionless).

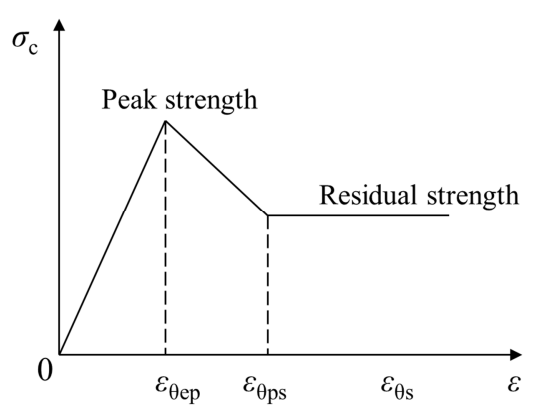

Figure 1. Simplified stress-strain curve.

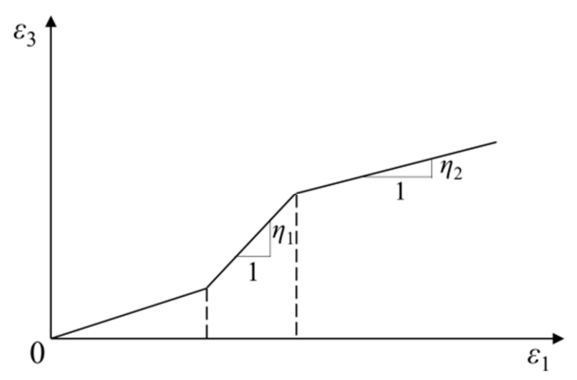

Figure 2. Simplified $\varepsilon_{1}-\varepsilon_{3}$ curve.

Many different yield criteria for rock materials have been proposed by many scholars at home and abroad in the context of the rapid development of rock mechanics, such as Mohr 
Coulomb criterion, Drucker Prager criterion, Griffith criterion, Hoek Brown criterion and twin shear strength criterion. Among them, Mohr Coulomb criterion can reflect the strength characteristics of geotechnical materials very well, and because its linear characteristics make the process and formula of analytical calculation more concise, it is used more widely. The Mohr Coulomb criterion can be rewritten as follows in polar coordinates:

$$
\left\{\begin{array}{l}
\sigma_{\theta}=K \sigma_{r}+\sigma_{c} \\
K=\frac{1+\sin \varphi}{1-\sin \varphi} \\
\sigma_{c}=\frac{2 C \cos \varphi}{1-\sin \varphi}
\end{array}\right.
$$

In this formula, $\sigma_{\theta}, \sigma_{r}$ are the surrounding rock's shear stress and radial stress, they correspond approximately to the maximum and minimum principal stresses; $\varphi$ is the friction angle of the rock; $C$ is the cohesion.

\subsection{Mechanical Model of Circular Cavern}

When the strain softening effect of rock mass is considered, there are multiple yield surfaces in the yield process. Therefore, when the load of surrounding rock reaches a certain state, the surrounding rock of tunnel can be divided into three zones from the inside to the outside, namely plastic residual zone, plastic softening zone and elastic zone, as shown in Figure 3. For the convenience of calculation, the smaller initial geostress is $P$, the larger is $\lambda P$, that is, the lateral pressure coefficient is $\lambda \geq 1$, and the polar axis direction of polar coordinate system is consistent with the direction of smaller in-situ stress. The uniform support pressure of the inner wall of the tunnel is $P_{i}$. When $\lambda>3$, tensile stress zone will appear in the surrounding rock, so the value range of lateral pressure coefficient is $1 \leq \lambda<3$.

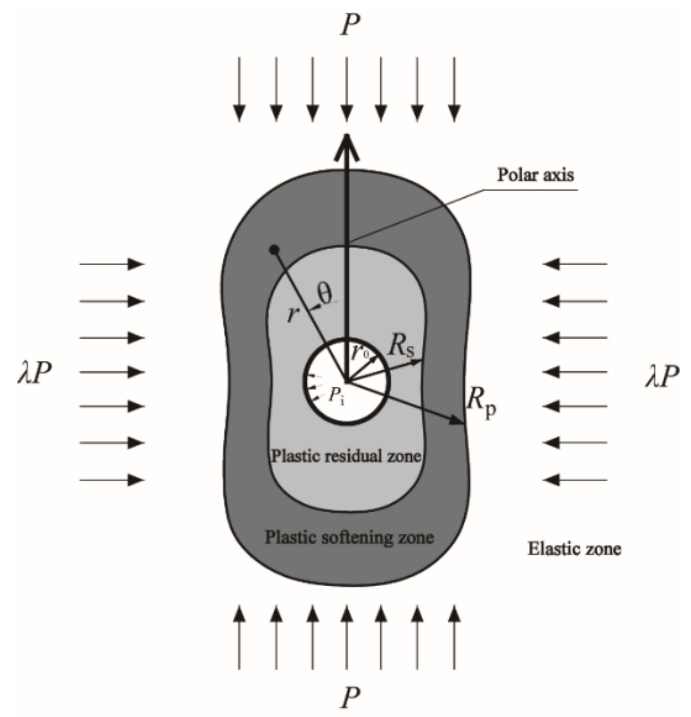

Figure 3. Mechanics model of circular tunnel.

\section{Analysis of Stress and Displacement in Elastic Zone}

\subsection{Solution of Stress Function in Elastic Zone}

Under non-axisymmetric load, many scholars' analytical calculations of the stress in the elastic zone are derived from the Lame solution, which is one of the main reasons for the error of the calculation results. In this paper, an appropriate stress function is found through trial algorithms, and a relatively accurate stress calculation formula in the elastic zone is obtained. The general formula of the stress function can be expressed as:

$$
\phi=\frac{1}{4}\left[\left(4 C_{4}-\frac{2 C_{1}}{r^{2}}+2 C_{2} r^{2}+C_{3} r^{4}\right) \cos 2 \theta+2\left(2 C_{5}+C_{7} r^{2}\right) \ln r+4 C_{8}+2 C_{6} r^{2}-C_{7} r^{2}\right]
$$

where $C_{1} \sim C_{8}$ are undetermined real constants, and the stress function naturally satisfies the coordination equation in the polar coordinate system. When physical strength is not taken into account, the stress component of the elastic zone represented by the stress function can be expressed as:

$$
\left\{\begin{array}{l}
\sigma_{r e}=\frac{1}{r} \frac{\partial \phi}{\partial r}+\frac{1}{r^{2}} \frac{\partial^{2} \phi}{\partial \theta^{2}} \\
\sigma_{\theta e}=\frac{\partial^{2} \phi}{\partial r^{2}} \\
\tau_{r \theta e}=-\frac{\partial}{\partial r}\left(\frac{1}{r} \frac{\partial^{2} \phi}{\partial \theta}\right)
\end{array}\right.
$$

At the elastoplastic interface, the rock mass obeys the Mohr-Coulumb yield criterion, and when $1 \leq \lambda<3$, the tangential and radial stresses have the following relationship:

$$
\sigma_{\theta e}+\sigma_{r e}=2 P+P(\lambda-1) \cos 2 \theta
$$

Combining (1) and (4), the stress boundary conditions on the elastoplastic interface are

$$
\left\{\begin{array}{l}
\left.\sigma_{r e}\right|_{r=R_{p}}=\frac{P[2+(\lambda-1) \cos 2 \theta]-\sigma_{c}}{1+K} \\
\left.\sigma_{\theta e}\right|_{r=R_{p}}=\frac{P K[2+(\lambda-1) \cos 2 \theta]+\sigma_{c}}{1+K} \\
\left.\tau_{r \theta e}\right|_{r=R_{p}}=0
\end{array}\right.
$$

It is known that the stress boundary condition at infinity of the surrounding rock is: 


$$
\left\{\begin{array}{l}
\left.\sigma_{r e}\right|_{r \rightarrow \infty}=0.5 P[1+\lambda-(\lambda-1) \cos 2 \theta] \\
\left.\sigma_{\theta e}\right|_{r \rightarrow \infty}=0.5 P[1+\lambda+(\lambda-1) \cos 2 \theta] \\
\left.\tau_{r \theta e}\right|_{r \rightarrow \infty}=P(\lambda-1) \cos \theta \sin \theta
\end{array}\right.
$$

Combining the above formulas, the general solution of the stress function $C_{1} \sim C_{8}$ can be obtained, and the stress analysis formula of the elastic zone in the surrounding rock from the stress function is:

$$
\begin{gathered}
\sigma_{r e}=\frac{1}{2} P(1+\lambda)-P(\lambda-1) \times\left[\frac{R_{p}{ }^{2}}{r^{2}}-\frac{R_{p}{ }^{4}}{2 r^{4}}+\left(\frac{1}{2}-\frac{R_{p}{ }^{2}}{r^{2}}+\frac{R_{p}{ }^{4}}{r^{4}}\right) \cos 2 \theta\right]+V_{1} \\
\sigma_{r e}=\frac{1}{2} P(1+\lambda)+\frac{1}{2} P(\lambda-1) \times\left[\left(1+\frac{2 R_{p}^{4}}{r^{4}}\right) \cos 2 \theta-\frac{R_{p}^{4}}{r^{4}}\right]-V_{1} \\
\tau_{r \theta e}=P(\lambda-1)\left(r^{2}-R_{p}{ }^{2}\right) \times\left[\left(r^{2}+2 R_{p}{ }^{2}\right) \cos 2 \theta-R_{p}{ }^{2}\right] \frac{\tan 2 \theta}{2 r^{4}}
\end{gathered}
$$

with

$$
V_{1}=P \frac{R_{p}{ }^{2}}{r^{2}} \frac{(3+K)(\lambda-1) \cos 2 \theta+2-2 K-2 \sigma_{c}}{2(1+K)}
$$

\subsection{Elastic Zone Displacement Solution}

Under non-axisymmetric load, the relationship between the displacement and stress of the elastic zone is,

$$
\begin{gathered}
\frac{\partial u_{e}}{\partial r}=\frac{1-\mu^{2}}{E} \sigma_{r e}-\frac{\mu+\mu^{2}}{E} \sigma_{\theta e} \\
\frac{u_{e}}{r}+\frac{1}{r} \frac{\partial v_{e}}{\partial \theta}=\frac{1-\mu^{2}}{E} \sigma_{\theta e}-\frac{\mu+\mu^{2}}{E} \sigma_{r e} \\
\frac{\partial v_{e}}{\partial r}+\frac{1}{r} \frac{\partial u_{e}}{\partial \theta}-\frac{v_{e}}{r}=\frac{2+2 \mu}{E} \tau_{r \theta e}
\end{gathered}
$$

Bring the calculated stresses into the above equations, using the radial and tangential displacements at infinity $\left(\left.u_{e}\right|_{r \rightarrow \infty}=0,\left.v_{e}\right|_{r \rightarrow \infty}=0\right)$, and the boundary conditions of the tangential displacements at the coordinate axis $(\theta=0, \pi / 2$, $\pi, 3 \pi / 2)$, the radial displacement of the elastic zone can be solved as:

$$
u_{e}=\frac{(1+\mu) R_{p}{ }^{2}}{6 E(1+K) r^{3}}\left(-V_{2}-V_{3}+V_{4}\right)+\frac{(1+\mu) R_{p}{ }^{2} \sigma_{c}}{E(1+K) r}
$$

with

$$
\begin{gathered}
V_{2}=P R_{p}^{2}(1+K)(\lambda-1) \quad(15) \\
V_{3}=6 r^{2} P[2-\mu-K \mu+\lambda(1+K)(\mu-1)] \quad \text { with } \quad u_{p}=r^{-\eta_{1}} R_{p}^{1+\eta_{1}} G \\
G=\frac{P(1+\mu)}{6 E(1+K)}\left[K-11+5 \lambda+5 K \lambda+6 \mu(1+K-\lambda-K \lambda)+(\lambda-1)(6 \mu+6 K \mu-13-7 K) \cos 2 \theta+\frac{6 \sigma_{c}}{P}\right]
\end{gathered}
$$

$$
V_{4}=P(\lambda-1)\left[2(1+K) R_{p}{ }^{2}+3 r^{2}(2 \mu+2 \mu K-3 K-5)\right] \cos 2 \theta
$$

\section{Analysis of Displacement, Stress and Range of Plastic Zone}

\subsection{Solution of Displacement Field in Plastic Zone}

Considering the dilatancy effect at the yield of the surrounding rock and assuming that the dilatancy gradient is constant, according to the plastic flow law, the radial and tangential strain of the rock in the plastic softening zone have the following relations:

$$
\eta_{1} \varepsilon_{\theta p}+\varepsilon_{r p}=0
$$

where $\eta_{1}$ is the dilatancy gradient of surrounding rock in the plastic softening zone, $\eta_{1} \geq 1$, when there is no dilatancy effect, $\eta_{1}=1 . \eta_{1}$ is obtained by the stress-strain test of the rock. As the diffuser gradient is greatly affected by the confining pressure, the average value of the maximum and minimum diffuser gradients can be taken.

Assuming that the strain in the plastic region is axisymmetric, the above equation can be written as

$$
\eta_{1} \frac{u_{p}}{r}+\frac{\partial u_{p}}{\partial r}=0
$$

Using the continuous condition of displacement on the elastoplastic interface, the displacement in the plastic softening zone is obtained as

Similarly, in the plastic residual region, the relation between radial and tangential strain can be expressed as

$$
\eta_{2} \varepsilon_{\theta s}+\varepsilon_{r s}=0
$$


where $\eta_{2}$ is the dilatancy gradient of surrounding rock in the plastic residual region. Similarly, the displacement in the plastic residual zone is obtained by using the plastic softening zone and the plastic residual zone to distinguish the continuous displacement conditions on the interface

$$
u_{s}=G \cdot r\left(\frac{R_{p}}{R_{s}}\right)^{1+\eta_{1}}\left(\frac{R_{s}}{r}\right)^{1+\eta_{2}}
$$

where $R_{\mathrm{s}}$ is the radius of the plastic residual zone.

\subsection{Solution of Stress Field in Plastic Zone}

Rock mechanics experiments and engineering practice show that the strain softening effect of rock mass is mainly caused by the reduction of cohesion of rock mass, and the internal friction angle changes little before and after yield, so the softening of the internal friction angle can be ignored. At this point, the softening modulus of rock mass strength is expressed as

$$
Q=\frac{\sigma_{c}-\sigma_{c s}}{\varepsilon_{\theta p s}-\varepsilon_{\theta e p}}=\frac{C-C_{s}}{\varepsilon_{\theta p s}-\varepsilon_{\theta e p}}
$$

where $\sigma_{c s}$ is the residual compressive strength of the rock mass, $C_{s}$ is the residual cohesion of the rock mass, $\varepsilon_{\theta p s}$ is the critical tangential strain of the rock mass from the plastic softening stage to the plastic residual stage, and $\varepsilon_{\theta e p}$ is the rock entering the plastic softening stage from the elastic stage Critical tangential strain. When the rock mass is an ideal elastoplastic medium, $Q=0$, and when the rock mass is an ideal brittle plastic medium, $Q \rightarrow \infty$, that is, as the brittleness of the rock mass increases, the softening modulus will gradually increase.

According to the trilinear stress-strain softening curve, the compressive strength of rock mass in the plastic softening stage is

$$
\sigma_{c p}=\sigma_{c}-G Q\left[\left(\frac{R_{p}}{r}\right)^{1+\eta_{1}}-1\right]
$$

The yield conditions of Mohr-Coulumb in the plastic softening zone can be expressed as

$$
\sigma_{\theta p}=K \sigma_{r p}+\sigma_{c p}
$$

Substituting it into the balanced differential equation, using the stress continuity condition on the elastoplastic interface $\left.\sigma_{r e}\right|_{r=R p}=\left.\sigma_{r p}\right|_{r=R p}$, the radial and tangential stresses in the plastic softening zone are obtained as

$$
\begin{gathered}
\sigma_{r p}=\left\{\frac{P[2+(\lambda-1) \cos 2 \theta]-\sigma_{c}}{1+K}+\frac{\eta_{1}\left(G Q+\sigma_{c}\right)+G Q+K \sigma_{c}}{(K-1)\left(K+\eta_{1}\right)}\right\}\left(\frac{R_{p}}{r}\right)^{1-K}+\frac{G Q}{K+\eta_{1}}\left(\frac{R_{p}}{r}\right)^{1+\eta_{1}}-\frac{G Q+\sigma_{c}}{K-1} \\
\sigma_{\theta p}=\frac{K G Q}{K+\eta_{1}}\left(\frac{R_{p}}{r}\right)^{1+\eta_{1}}-\frac{K\left(G Q+\sigma_{c}\right)}{K-1}+\sigma_{c}+K\left\{\frac{P[2+(\lambda-1) \cos 2 \theta]-\sigma_{c}}{1+K}+\frac{\eta_{1}\left(G Q+\sigma_{c}\right)+G Q+K \sigma_{c}}{(K-1)\left(K+\eta_{1}\right)}\right\} \times\left(\frac{R_{p}}{r}\right)^{1-K}-G Q\left[\left(\frac{R_{p}}{r}\right)^{1+\eta_{1}}-1\right]
\end{gathered}
$$

In the plastic residual zone, the Mohr-Coulumb yield condition can be expressed as,

$$
\sigma_{\theta s}=K \sigma_{r s}+\sigma_{c s}
$$

Bringing it into the balanced differential equation, using the boundary condition $\left.\sigma_{r s}\right|_{r=r_{0}}=P_{i}$, when the cave wall is under uniform support pressure, and the radial and tangential stresses in the plastic residual zone are obtained as

$$
\begin{gathered}
\sigma_{r s}=\frac{(K-1) P_{i}+\sigma_{c s}}{K-1}\left(\frac{r_{0}}{r}\right)^{1-K}+\frac{\sigma_{c s}}{1-K} \\
\sigma_{\theta s}=K \frac{(K-1) P_{i}+\sigma_{c s}}{K-1}\left(\frac{r_{0}}{r}\right)^{1-K}+\frac{K \sigma_{c s}}{1-K}+\sigma_{c s}
\end{gathered}
$$

\subsection{Calculation of Plastic Zone}

According to the definition of softening modulus of rock mass strength,

$$
\varepsilon_{\theta p s}-\varepsilon_{\theta e p}=\frac{\sigma_{c}-\sigma_{c s}}{Q}
$$

where $\varepsilon_{\theta p s}$ and $\varepsilon_{\theta e p}$ can be calculated by (17) and (20),

$$
\begin{gathered}
\varepsilon_{\theta e p}=G \\
\varepsilon_{\theta p s}=G\left(\frac{R_{p}}{R_{s}}\right)^{1+\eta_{1}}
\end{gathered}
$$

Combine the above 3 equations to get:

$$
\frac{R_{p}}{R_{s}}=\left(\frac{\sigma_{c}-\sigma_{c s}}{G Q}+1\right)^{\frac{1}{1+\eta_{1}}}
$$

At the same time, using the plastic softening zone and plastic residual to distinguish the stress continuity condition on the interface can be obtained:

$$
\frac{r_{0}}{R_{s}}=\left[\frac{(K-1) V_{5}}{(K-1) P_{i}+\sigma_{c s}}\right]^{\frac{1}{1-K}}
$$

with

$$
\omega=R_{p} / R_{s}
$$




$$
V_{5}=\omega^{1+\eta_{1}} \frac{G Q}{K+\eta_{1}}-\frac{G Q+\sigma_{c}}{K-1}+\frac{\sigma_{c s}}{K-1}+\omega^{1-K} \times\left\{\frac{P[2+(\lambda-1) \cos 2 \theta]-\sigma_{c}}{1+K}+\frac{\eta_{1}\left(G Q+\sigma_{c}\right)+G Q+K \sigma_{c}}{(K-1)\left(K+\eta_{1}\right)}\right\}
$$

In combination with equation (35) and Equation (36), the boundary formula of plastic residual zone and plastic softening zone can be expressed as,

$$
\begin{gathered}
R_{p}=r_{0}\left(\frac{\sigma_{c}-\sigma_{c s}}{G Q}+1\right)^{\frac{1}{1+\eta_{1}}}\left[\frac{(K-1) V_{5}}{(K-1) P_{i}+\sigma_{c s}}\right]^{\frac{1}{K-1}} \\
R_{s}=r_{0}\left[\frac{(K-1) V_{5}}{(K-1) P_{i}+\sigma_{c s}}\right]^{\frac{1}{K-1}}
\end{gathered}
$$

When $r=r_{0}$, the radial displacement on the cave wall can be obtained as,

$$
u_{0}=G \cdot r_{0}\left(\frac{R_{p}}{R_{s}}\right)^{1+\eta_{1}}\left(\frac{R_{S}}{r_{0}}\right)^{1+\eta_{2}}
$$

In fact, when deviates from 1 , the strain in the plastic zone is only approximately axisymmetric on the side with larger ground stress $(\lambda P)$, so the displacement calculated by the above formula is closest to the true value only if $\theta$ is at $90^{\circ}$ and $270^{\circ}$, and the error is relatively large on the side where the ground stress is smaller $(\lambda P)$. The finite element calculation results of a large number of circular cavities show that when the plastic zone is large $\left(R_{\mathrm{pmin}}>1.5 r_{0}\right)$, the ratio of the radial

displacement of any point on the cavity wall to its minimum value is approximately calculated as follows,

$$
\frac{u_{0}}{\left.u_{0}\right|_{\theta=\pi / 2}}=\sqrt{\lambda} \cos ^{2} \theta+\sin ^{2} \theta
$$

At this time, the approximate solution of the radial displacement of the circular cavity can be obtained as follows,

$$
u_{0}=r_{0} M\left(\sqrt{\lambda} \cos ^{2} \theta+\sin ^{2} \theta\right) \alpha^{1+\eta_{1}} \beta^{1+\eta_{2}}
$$

with

$$
\begin{gathered}
M=\frac{P(1+\mu)}{E(1+K)}\left[\frac{\sigma_{c}}{P}-K-4+3 \lambda+2 K \lambda-2 \mu(1+K)(\lambda-1)\right] \\
\alpha=\left(\frac{\sigma_{c}-\sigma_{c s}}{M Q}+1\right)^{\frac{1}{1+\eta_{1}}} \\
\beta=\left[\frac{(K-1) V_{5}}{(K-1) P_{i}+\sigma_{c s}}\right]^{\frac{1}{1-K}}
\end{gathered}
$$

The calculation analysis shows that when $\lambda=1$, the above formula is the solution of literature [16], that is, the analytical solution considering strain softening and dilatancy under axisymmetric loads is a special case of this approximate solution. In the calculation process, the stress state of surrounding rock should be determined. If the surrounding rock has not entered the plastic residual stage, the value of $\alpha$ will be 1 . If the surrounding rock has entered the plastic softening stage, the displacement will be calculated using Equation (14).

\section{Calculation Examples and Analysis}

For a circular tunnel with a radius of $r_{0}=2.965 \mathrm{~m}$, ignoring the disturbance of the surrounding rock during the excavation process, the physical as well as mechanical parameters of the surrounding rock are: elastic modulus $E=20 \mathrm{GPa}$, Poisson ratio $\mu=0.3$, and the friction angle in the rock body $\varphi=30^{\circ}$, cohesion $C=C_{\mathrm{s}}=0.3 \mathrm{MPa}$, softening modulus $Q=0$, expansion gradient $\eta_{1}=\eta_{2}=1$, lower ground stress level is $10 \mathrm{MPa}$, lateral pressure coefficient $\lambda$ is $1,1.5,2$. The stress field, plastic zone radius and radial displacement of the surrounding rock of the tunnel under different lateral pressure coefficients were calculated, and compared with the existing analytical solutions and finite element solution (Phase2D).

Table 1. Calculation and comparison of the ratio of displacement and tunnel radius $u_{0} / r_{0}$.

\begin{tabular}{llllr}
\hline \multirow{2}{*}{$\lambda$} & \multicolumn{2}{l}{ Finite element solutions } & \multicolumn{2}{l}{ Solutions in this paper } \\
\cline { 2 - 5 } & $\boldsymbol{\theta}=\mathbf{0}^{\circ}$ & $\boldsymbol{\theta}=\mathbf{9 0}{ }^{\circ}$ & $\boldsymbol{\theta}=\mathbf{0}^{\circ}$ & $\boldsymbol{\theta}=\mathbf{9 0}$ \\
\hline 1 & $0.13 \%$ & $0.13 \%$ & $0.13 \%$ & $0.13 \%$ \\
1.5 & $0.29 \%$ & $0.24 \%$ & $0.34 \%$ & $0.28 \%$ \\
2 & $0.47 \%$ & $0.34 \%$ & $0.44 \%$ & $0.31 \%$ \\
\hline
\end{tabular}

The calculation results show that the stress fields in the elastic zone and the plastic zone of the surrounding rock obtained in this paper are very close to the finite element calculation results in terms of distribution form and value. From Table 1 and Table 2, it can be seen that compared with the analytical solutions of Cai and Sun, the $R_{\mathrm{p}} / r_{0}$ calculated by the approximate solution given in this paper is closer to the finite element calculation result and slightly larger than the finite element solution. When the lateral pressure coefficient $\lambda=1 \sim 2$ and the plastic zone is relatively large, the approximate solution of cave wall displacement obtained in this paper is 
also very close to the finite element solution.

Table 2. Calculation and comparison of the ratio of plastic zone radius and tunnel radius $R p / r_{0}$.

\begin{tabular}{|c|c|c|c|c|c|c|c|c|}
\hline \multirow{2}{*}{$\lambda$} & \multicolumn{2}{|c|}{ Solutions of Cai } & \multicolumn{2}{|c|}{ Solutions of Sun } & \multicolumn{2}{|c|}{ Finite element solutions } & \multicolumn{2}{|c|}{ Solutions in this paper } \\
\hline & $\theta=0^{\circ}$ & $\theta=90^{\circ}$ & $\theta=0^{\circ}$ & $\theta=90^{\circ}$ & $\theta=0^{\circ}$ & $\theta=90^{\circ}$ & $\theta=0^{\circ}$ & $\theta=90^{\circ}$ \\
\hline 1 & 3.18 & 3.18 & 3.18 & 3.18 & 3.04 & 3.04 & 3.18 & 3.18 \\
\hline 1.5 & 4.16 & 2.78 & 4.22 & 2.85 & 3.37 & 2.36 & 3.54 & 2.78 \\
\hline 2 & 4.96 & 2.30 & 5.11 & 2.62 & 3.78 & 2.02 & 3.86 & 2.30 \\
\hline
\end{tabular}

From Table 3, we can know that the lateral pressure coefficient has a great influence on the displacement value of the cave wall, and with the increase of the softening modulus and expansion gradient of the surrounding rock, the displacement of the cave wall will increase significantly, showing the strain softening and expansion of the surrounding rock has a great influence on the displacement field of the surrounding rock. Thus, it is necessary to consider the softening and dilatancy effect of the surrounding rock when analyzing the elastic-plastic analysis of the surrounding rock, especially when analyzing the deformation and plasticity area of the surrounding rock in the extruded stratum, so as to get a safer conclusion.

Table 3. Calculation of the ratio of displacement and tunnel radius $u 0 / r 0$ with different soften parameter and dilatancy grads.

\begin{tabular}{|c|c|c|c|c|c|c|}
\hline \multirow{2}{*}{$\lambda$} & \multicolumn{2}{|c|}{$\mathrm{C}_{\mathrm{s}}=0.3 \mathrm{MPa}, \mathrm{Q}=0, \eta_{1}=1, \eta_{2}=1$} & \multicolumn{2}{|c|}{$C_{s}=0.1 \mathrm{MPa}, Q=1 \mathrm{GPa}, \eta_{1}=1.5, \eta_{2}=1.2$} & \multicolumn{2}{|c|}{$C_{s}=0.1 \mathrm{MPa}, Q=50 \mathrm{GPa}, \eta_{1}=2, \eta_{2}=1.5$} \\
\hline & $\theta=0^{\circ}$ & $\theta=90^{\circ}$ & $\theta=0^{\circ}$ & $\theta=90^{\circ}$ & $\theta=0^{\circ}$ & $\theta=90^{\circ}$ \\
\hline 1 & $0.13 \%$ & $0.13 \%$ & $1.36 \%$ & $1.36 \%$ & $2.25 \%$ & $2.25 \%$ \\
\hline 1.5 & $0.34 \%$ & $0.28 \%$ & $3.09 \%$ & $2.52 \%$ & $4.89 \%$ & $3.99 \%$ \\
\hline 2 & $0.44 \%$ & $0.31 \%$ & $3.63 \%$ & $2.57 \%$ & $5.39 \%$ & $3.81 \%$ \\
\hline
\end{tabular}

\section{Conclusion}

The ground stress lateral pressure coefficient of the surrounding rock of circular tunnel has a great influence on its plastic zone, stress field and displacement field, and the analytical method research of the elastic-plastic analysis of the surrounding rock of circular tunnel is mostly on the basis of the axially symmetric distribution of load, which affects its application in engineering practice. Considering the yield of the strain softening and expansion of rock mass effect, using three linear elastic - plastic softening - plastic residual stress and strain softening model and the Mohr-Coulomb yield criterion, the axisymmetric load was deduced under the circular tunnel surrounding rock elastic zone and the plastic softening zone and plastic zones in the surrounding of residual stress field, strain field, displacement field and plastic zone radius of the approximate analytical solution, suitable for plastic zone of surrounding rock is bigger $\left(R_{\text {pmin }}>1.5 r_{0}\right)$ and the lateral pressure coefficient is $1 \leq \lambda<3$. The approximate analytical method is close to the calculation result of finite element method and can replace the finite element method to carry out simple elastic-plastic analysis of surrounding rock.

\section{References}

[1] Jiang MJ, Shen ZJ. The expansion of linear softening cylindrical hole with dilatation is considered [J]. Chinese Journal of Rock Mechanics and Engineering, 1997, 16 (6): 550-557.

[2] Sharan SK. Exact and approximate solutions for displacements around circular openings in elastic-brittle-plastic HoekBrown rock [J]. International Journal of Rock Mechanics \&
Mining Sciences, 2005, 42: 542-529.

[3] Yu MH. Advances in strength theory of materials under complex stress state in the 20th century [J]. Applied Mechanics Reviews, 2002, 55 (3): 169-218.

[4] Fan W, Yu MH, Chen LW, et al. Unified Elastoplastic Solution for Surrounding Rocks of Openings with Consideration of Material Dilatancy and Softening [J]. Chinese Journal of Rock Mechanics and Engineering, 2004, 23 (19): 3213-3220.

[5] Yu XF, Zheng Y. Stability analysis of surrounding rock of underground engineering [M]. Beijing: China Coal Industry Publishing House, 1983.

[6] Sun GZ. Rock mass mechanics foundation [M]. Beijing: Science Press, 1983.

[7] Cai XH, Cai YP. Calculation of structural stress of hydraulic pressure tunnel [M]. Beijing: China Water Power Press, 2004.

[8] Park KH, Kim YJ. Analytical solution for a circular opening in an elastic-brittle-plastic rock [J]. International Journal of Rock Mechanics \& Mining Sciences, 2006, 43: 616-622.

[9] Kargar AR. An analytical solution for circular tunnels excavated in rock masses exhibiting viscous elastic-plastic behavior [J]. International Journal of Rock Mechanics and Mining Sciences, 2019, 124: 104-128.

[10] Wang SL, Wu ZJ, Guo MW, Ge X. Theoretical solutions of a circular tunnel with the influence of axial in situ stress in elastic-brittle-plastic rock [J]. Tunn Undergr Space Technol, 2012, 30: 155-168.

[11] Zhou XP, Li JL. Hoek-Brown criterion applied to circular tunnel using elastoplasticity and in situ axial stress $[\mathrm{J}]$. Theor Appl Fract Mech, 2011, 56: 95-103.

[12] Carranza-Torres, C., Fairhurst, C. The elasto-plastic response of underground excavations in rock masses that satisfy the Hoek-Brown failure criterion [J]. Int. J. Rock. Mech. Min. Sci, 1999, 36: 777-809. 
[13] R. Jimenez, A. Serrano, C. Olalla. Linearization of the Hoek and Brown rock failure criterion for tunnelling in elasto-plastic rock masses [J]. International Journal of Rock Mechanics and Mining Sciences, 2008, 45 (7) 1153- 1163.

[14] Sharan S. Elastic-brittle-plastic analysis of circular openings in Hoek-Brown media [J]. Int J Rock Mech Min Sci. 2003; 40 (6): 817-824
[15] Lu AZ, Zhang XL, Wang SJ. Analytic method for elasto-plastic analysis of circular tunnels under non-axisymmetric stresses [J]. Chin J Rock Mech Eng, 2018, 37: 14-22.

[16] Ma SJ. The softening deformation analysis and stimulation calculation of dilatancy in the soft rock tunnel [D]. Shenyang: Liaoning Technical University, 2001. 